\title{
Procesos de enseñanza de la función exponencial. Un acercamiento cualitativo
}

Fecha de recepción : 2020-02-17 • Fecha de aceptación: 2020-05-21 • Fecha de publicación: 2020-09-10

\author{
Carlos Armando Tarira Caice ${ }^{1}$ \\ Unidad Educativa Julio Moreno Espinosa, Ecuador \\ ctariracaice@hotmail.com \\ https://orcid.org/0000-0002-2139-186X
}

Hugo Parra-Sandoval ${ }^{2}$

Universidad del Zulia, Venezuela

hps1710@gmail.com

https://orcid.org/0000-0002-4484-2619

Mercedes Delgado González ${ }^{3}$

Universidad del Zulia, Venezuela

merdelgon@gmail.com

https://orcid.org/0000-0002-4292-8339

\section{Resumen}

El estudio de la función exponencial, como objeto matemático de enseñanza, es de suma importancia para la comprensión de actividades humanas vinculadas a diversos campos, tales como economía, biología, física, medicina o demografía. Entre ellas se puede destacar el estudio del crecimiento y decrecimiento exponencial, tema que se enriquece con su construcción gráfica o geométrica en un plano ortogonal, además permite manejar variables físicas tales como el tiempo respecto a otras como la población, o la radiactividad. Esta investigación pretende describir el proceso de enseñanza de la función exponencial mediante un acercamiento cualitativo. La metodología utilizada fue cualitativa y se enmarcó en un estudio de caso. El informante fue un docente de matemática; se realizó una descripción del proceso de enseñanza de este tema en una unidad educativa ecuatoriana. Los resultados muestran que el docente usa una terminología matemática adecuada, su actuación es de tipo 
expositivo magistral, establece un tipo de conexión interdisciplinar muy sucinto y tiene estructurado un plan de trabajo del cual no se desvía. Entre las conclusiones se destacan que la forma tradicional conductista de realizar la praxis pedagógica aún se puede apreciar en Ecuador, caracterizada por el trabajo mecánico y repetitivo al enseñar conocimientos específicos de la materia.

\title{
Palabras clave: procesos de enseñanza, función exponencial, matemática, enseñanza de la función exponencial, cuarteto de conocimiento.
}

\begin{abstract}
The study of exponential function as a mathematical object of teaching is of utmost importance for the understanding of human activities related to various fields, such as economics, biology, physics, medicine or demography. Among them we can highlight the study of exponential growth and decrease, a topic that is enriched by its graphic or geometric construction on an orthogonal plane. It also allows managing physical variables such as time relative to others such as population, or radioactivity. This research aims to describe the process of teaching exponential function through a qualitative approach. The methodology used was qualitative. It was framed in a case study. The informant was a teacher of mathematics; a description was made of the teaching process on this topic in an Ecuadorian educational unit. The results show that the teacher uses adequate mathematical terminology, his performance is of a masterly expository type, establishes a very succinct type of interdisciplinary connection and has a structured work plan from which he does not deviate. Among the conclusions, it should be noted that the traditional behaviorist way of carrying out pedagogical praxis can still be appreciated in Ecuador, characterized by mechanical and repetitive work when teaching specific knowledge of the subject.
\end{abstract}

Keywords: teaching processes, exponential function, mathematics, teaching of exponential function, knowledge quartet. 


\section{Introducción}

La práctica docente de la matemática en el aula se ha visto fuertemente cuestionada por investigaciones, y la sociedad en general, en función de los resultados de procesos educativos. Actualmente se discute sobre la calidad de la práctica docente y de la educación, entendiendo a esta última como el grado de cercanía entre lo establecido en los fines del sistema educativo nacional y el logro de la población estudiantil (Gómez, 2007).

En general, la práctica docente ha sido influenciada por reformas curriculares y corrientes teóricas que promueven nuevas formas sobre cómo desarrollar dicha experiencia. Según Castro, PinoFan y Parra-Urrea (2018), por más de tres décadas la comunidad de educadores de matemática ha estado interesada en describir, analizar y determinar el conocimiento requerido de esta ciencia para lograr procesos de enseñanza que garanticen el aprendizaje de sus estudiantes.

Por otra parte, la praxis pedagógica tradicional se caracteriza, según Avendaño (2013), por el trabajo mecánico y repetitivo al enseñar conocimientos específicos. En el caso de Ecuador esta praxis pedagógica está muy presente, así lo evidencian investigaciones como las de Bravo, Trelles y Barrazueta (2017) y Suárez (2019), además de la experiencia como docente del investigador en aulas de la Educación del Bachillerato General Unificado, independientemente de la formación que el educando haya tenido.

El empleo de una metodología tradicional formal del enfoque conductista por parte de los profesores es, en la gran mayoría de las veces, resultado de su propia experiencia como estudiante y, en el mejor de los casos, de su juicio sobre cómo las personas aprenden y se apropian de los conocimientos. La mayor parte de los docentes se caracterizan por tener habilidades y conocimientos para transmitir los contenidos que le son proporcionados a través de libros o documentos oficiales como los programas, pero sin poder ir más allá en la noción, sin conocer las razones de la existencia de tal o cual saber, tanto en la historia misma de la disciplina como en el currículo escolar.

De acuerdo a Parra (2013), Garii y Silverman (2009) y Monaghan (2007) la contextualización de las matemáticas por parte del profesor no es fácil y mucho menos automática, muy a pesar de que esta asignatura han sido producto de la cultura humana para satisfacer sus necesidades materiales y espirituales, los docentes tienen una capacidad limitada para contextualizar el conocimiento y deficiencias para vincular lo que imparten con otras disciplinas u otros niveles educativos, a pesar que "el pensamiento matemático se desarrolla en todos los seres humanos en el enfrentamiento cotidiano a sus múltiples tareas" (Cantoral, Farfán, Cordero, Alanís y Garza 2003:19).

Luego, desde la experiencia del investigador principal, sólo algunos docentes cuentan con conocimientos didácticos para enseñar los contenidos eficazmente, pero no los ponen en práctica. Al mismo tiempo carecen de otros, como la utilidad y necesidad de la planeación, empleo y variación de estrategias pedagógicas activas para el proceso de enseñanza y aprendizaje o la utilización de recursos. Se caracterizan más que nada por el hecho de poseer las bases matemáticas indispensables para comprender y profundizar en los contenidos. 
Esta situación se ha visto con mayor énfasis en Ecuador a partir de la última década, principalmente desde que otros profesionales ajenos al área de la docencia, tales como ingenieros, administradores, contadores u otros han ingresado a las instituciones educativas como profesores de matemática, sin tener una adecuada formación docente.

En este sentido, mientras que unos están más especializados en el saber, otros lo están, pero en un aspecto pedagógico, provocando que la intencionalidad de la enseñanza sea diferente. Por lo general, son pocos los profesores que tienen ambos conocimientos y saben integrarlos. Esto ha traído como consecuencia que los estudiantes no vinculen los temas estudiados en matemática con su cotidianidad, obteniendo así aprendizajes descontextualizados y sin sentido.

Por otro lado, los temas matemáticos, y en particular las funciones exponenciales, son de suma importancia para la comprensión de las actividades humanas, vinculadas a diversos campos tales como la economía, la biología, la física, la medicina o la demografía; sin embargo, son presentados sin vinculación alguna con estas áreas del conocimiento. En Ecuador encontramos inconvenientes en la forma de abordarlo por parte de algunos docentes; habitualmente es a través de la solución de ejercicios, elaboración de tablas de valores, realización de gráficas y estudio de la misma, este proceso requiere la inversión de mucho tiempo, dejando poco espacio para el análisis, las reflexiones y sus aplicaciones a las actividades humanas, trayendo como consecuencia un alto grado de dificultad por parte de los estudiantes para la comprensión de esta unidad curricular tan importante.

Con relación al conocimiento del contenido matemático por parte de los profesores, Rowland et al. (2005), realizaron un estudio sobre este conocimiento de profesores en formación de educación primaria del Reino Unido, para ello analizaron sesiones de clases de algunos educadores. Su objetivo fue localizar las formas en que se funda el conocimiento y la enseñanza del docente de matemática. El análisis de los datos obtenidos condujo a la identificación de un cuarteto de conocimiento, con cuatro categorías o unidades a través de los cuales se pueden observar los conocimientos matemáticos y didácticos puestos en práctica de los profesores. De esta forma se origina el modelo del cuarteto de Conocimiento (The Knowledge Quartet - KQ) cuyas categorías o unidades se denominan: fundamento, transformación, conexión y contingencia, tal como se muestran en la Figura 1.

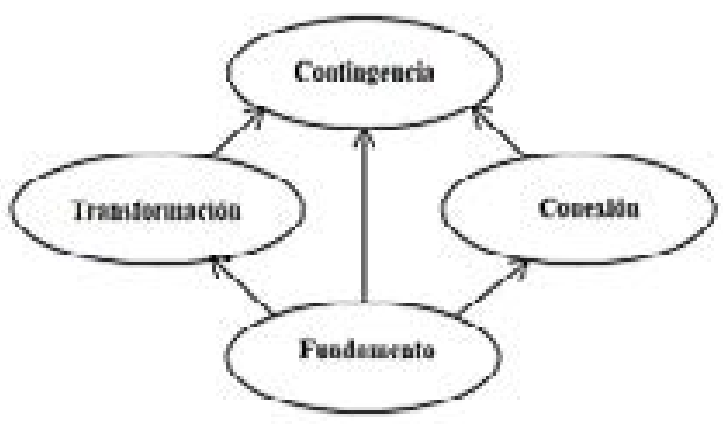

Figura 1. Modelo el cuarteto de Conocimiento

Fuente: Rowland et al. (2005) 
A continuación, se describen cada una de las categorías según Rowland et al. (2005):

Categoría 1. Fundamento. Se refiere al conocimiento, la comprensión per se y el saber necesario para enseñar matemáticas, así como las creencias sobre la naturaleza de esta ciencia. Las finalidades de la educación matemática y las condiciones sobre las cuales los alumnos aprenderán mejor también contempla el aprendizaje de los docentes, en la preparación intencionadamente o no, de su rol en el aula. Las componentes clave de este marco conceptual son: el conocimiento y la comprensión de las matemáticas, esta categoría coincide con lo que Shulman (1987) Ilama comprensión y lo relaciona con el conocimiento didáctico del contenido.

Categoría 2. Transformación. Este se basa en la observación propuesta por Shulman (1987) de los conocimientos para la enseñanza, caracterizados por la capacidad de un docente para transformar el conocimiento que posee de manera que sean pedagógicamente poderosos. Esta caracterización se pone de manifiesto en la redacción que distingue entre saber algo de matemáticas para sí mismo, con la finalidad de poder ayudar a otra persona a aprenderlo. Como indica Shulman (1986), el exponer ideas a los alumnos implica representarlas en forma de analogías, ilustraciones, ejemplos, explicaciones y demostraciones. Esto incluye el uso de ejemplos para ayudar a la formación de conceptos para demostrar los procedimientos, y la selección de actividades para los estudiantes.

Categoría 3. Conexión. Se refiere a la coherencia en la planificación o la enseñanza que se muestra a través de un episodio, una clase o una serie de clases. La Matemática es notable por su relación como un cuerpo de conocimiento y un campo de investigación. La mente del maestro y su gestión a través del discurso matemático en el aula, la concepción de la coherencia incluye la secuenciación de los temas de instrucción dentro y entre las clases, incluyendo el orden de tareas y ejercicios. En gran medida, estos reflejan las decisiones que implican no sólo el conocimiento de las conexiones estructurales dentro de las matemáticas mismas, sino también el conocimiento de las demandas cognitivas relativas a diferentes temas y tareas.

Parra-Sandoval (2018) propone cinco tipos de conexiones: intraconceptual, interconceptual, temporal, interdisciplinar y extracurricular. Las intraconceptuales, se refieren a las conexiones asociadas a un mismo concepto matemático. Las interconceptuales son aquellas relaciones que se establecen entre diferentes conceptos matemáticos y las temporales se establecen entre los diferentes conceptos matemáticos trabajados de acuerdo a los niveles educativos; es decir, con los conceptos matemáticos anteriores y posteriores a él, presentes en el currículo escolar.

La conexión interdisciplinaria significa la relación de un concepto matemático con otras disciplinas del currículo, mientras que las extracurriculares hacen referencia a las conexiones de los conceptos matemáticos con aquellos conocimientos propios de una comunidad de prácticos (conocimientos funcionales) y los conocimientos cotidianos.

Categoría 4. Contingencia. Se refiere a lo que es casi imposible de planificar. Los dos componentes que constituyen esta categoría se derivan de los datos y son la preparación para responder a las ideas de los niños y la preparación en caso de tener que desviarse de la planificación. Shulman (1987) plantea que la enseñanza generalmente comienza a partir de 
algún tipo de texto, un programa de estudios, en última instancia, una secuencia de acciones planificadas, destinados a ser llevado a cabo por el profesor dentro de una lección o unidad de algún tipo. Mientras que el estímulo para la enseñanza de los profesores puede ser planificado, algunas de las respuestas de los estudiantes no se pueden prever.

El marco conceptual de Rowland et al. (2005) introduce un instrumento útil para observar, describir y reflexionar sobre cómo el conocimiento matemático y didáctico del profesor de matemática entra en juego en el aula, es decir, cómo lo implementa en su práctica de enseñanza.

Con relación a lo anteriormente expuesto se realizó una investigación que condujo a este artículo, el cual tiene como objetivo describir el proceso de enseñanza de la función exponencial mediante un acercamiento cualitativo; para ello se estableció una vinculación con el modelo del cuarteto de conocimiento propuesto por Rowland et al. (2005).

\section{Metodología}

El presente estudio se realizó mediante un enfoque cualitativo, y su carácter inductivo permitió al investigador el desarrollo de conceptos, categorías y comprensiones partiendo de la información obtenida en el escenario. En tal sentido, la pretensión no fue generalizar los hallazgos, sino lograr describir los procesos de enseñanza de la función exponencial en una institución educativa de Ecuador.

La investigación está enmarcada en un estudio de caso, con una dimensión descriptiva e interpretativa, debido a que se describen, caracterizan o identifican los distintos hechos relacionados con su campo observacional o componente empírico, como es la descripción del proceso de enseñanza de la función exponencial. Desde la perspectiva interpretativa, su finalidad es interpretar y teorizar sobre el fenómeno estudiado, en este sentido, se desarrollan categorías conceptuales para ilustrar o defender presupuestos teóricos, a partir de un análisis inductivo (Husén, 1988).

De acuerdo con lo analizado se observó un caso (un profesor de matemática) y su clase correspondiente al tema función exponencial. Este método permitió observar las características del docente en el contexto de su aula, con el propósito de analizar distintos aspectos de un mismo fenómeno. Nunca hubo la pretensión de alcanzar conclusiones generalizables (Martínez, 2006), sino mostrar en su ambiente natural del aula la praxis pedagógica utilizada cuando desarrolló el tema de las funciones exponenciales para ser analizada en profundidad.

Para el artículo participó un docente de matemática, quien manifestó su disposición. Este profesor pertenece a la planta docente de una Unidad Educativa de Ecuador, cuya modalidad es presencial de jornada matutina y vespertina con nivel educativo de inicial; educación básica y bachillerato, ubicada en la zona: Urbana INEC y cuenta con 3097 estudiantes aproximadamente.

\section{Fases del trabajo de campo o ejecución}


El trabajo de campo para el desarrollo de la investigación se realizó en dos fases de acuerdo al objetivo planteado.

Fase 1. Inicialmente se realizó un conversatorio con el docente en cuanto a la posibilidad de observar sus prácticas de enseñanza con relación al tema funciones exponenciales. Luego de conseguir su aprobación se procedió a entrar a sus clases como observador; antes de iniciar las grabaciones para el registro de información se hizo el ingreso para familiarizar con el docente y los estudiantes, de esta manera se fomenta un clima de confianza, posteriormente se grabaron las clases correspondientes al tema.

Fase 2. Descripción del proceso de enseñanza de la función exponencial. Para esto se utilizó la técnica de observación no participante; se trascribieron y analizaron las grabaciones obtenidas, es decir, el análisis se realizó después del trabajo de campo, lo cual según Quintana y Montgomery (2006), concierne principalmente al desarrollo del sistema de codificación.

Para esto se realizó el desarrollo del sistema de categorías apriorísticas presentado en la Tabla 1 , el cual constituyó el primer paso para la estructuración de la descripción tras la culminación del trabajo de campo. Es de advertir que estas categorías y propiedades fueron provisionales, porque este trabajo constituye una primera entrada y, por tanto, no pueden ser categorías y propiedades definitivas, las mismas pueden cambiar durante el desarrollo de otras investigaciones posteriores.

Tabla 1.

Sistema de categorías con relación al objetivo.

\begin{tabular}{ll}
\hline CATEGORÍA & PROPIEDADES \\
\hline Fundamento & Claridad de los objetivos \\
& Recurrencia al programa o libro texto \\
& Concentración en los procedimientos \\
& Exhibición de conocimiento del tema \\
& Uso de la terminología matemática \\
\hline Transformación & Selección de ejemplos \\
& Selección de representaciones \\
& Uso del material de enseñanza \\
& Demostración para enseñar un procedimiento \\
\hline Conexión & Coherencia entre la planificación y la clase \\
& Conexiones de la Función exponencial con otras disciplinas \\
& (interdisciplinaria) \\
& Conexiones entre procedimientos (interconceptual) \\
& Conexiones sobre conceptos \\
& Reconocimiento de la adecuación conceptual \\
\hline Contingencia & Desvío del plan de trabajo \\
& Responde a las ideas del alumno \\
& Uso de oportunidades \\
& Percepción del profesor durante la clase \\
\hline
\end{tabular}

Fuente: elaboración propia (2020)

La información necesaria para la realización del estudio se recolectó a través de videograbaciones y las transcripciones de las clases a las cuales se hizo acompañamiento por medio de la 
observación no participante.

El análisis e interpretación de los datos cualitativos se realizó en función a las diferentes categorías con sus respectivas propiedades expresadas en la Tabla 1, atendiendo al objetivo formulado en la investigación. Esto permitió hacer manejable el cúmulo de información recogida y presentar los resultados en función del objetivo. El proceso seguido fue el siguiente:

Para la categoría Fundamento se observó si el docente participante tenía claridad de los objetivos que debía cumplir según la programación o planificación; la recurrencia al programa o libro texto como apoyo, la concentración en los procedimientos, su exhibición de conocimiento del tema y el uso de la terminología matemática que él hace durante la clase.

Con relación a la categoría Transformación se hizo énfasis en describir la selección de ejemplos, selección de representaciones, uso del material de enseñanza y la demostración para enseñar un procedimiento por parte de este docente.

Mientras que en la Conexión fue basada en ver la coherencia entre la planificación realizada por el docente y la clase dada, las conexiones de la función exponencial con otras disciplinas, los vínculos entre procedimientos, entre conceptos y el reconocimiento de la adecuación conceptual por parte del docente.

Por último, para la categoría Contingencia se observó la preparación para responder cuando ocurre por alguna causa un desvío del plan de trabajo, cómo responde a las ideas del alumno, el uso de oportunidades y la percepción del profesor durante la clase.

\section{Resultados}

Para la descripción del proceso de enseñanza de la función exponencial de este docente se estableció una vinculación con el modelo del cuarteto de Conocimiento propuesto por Rowland et al. (2005), a través de este se pudo observar los conocimientos matemáticos y didácticos puestos en práctica mediante el apoyo del sistema de categorías de la Tabla 1.

Antes de entrar en detalle con cada categoría, es pertinente hacer una descripción general del proceso de enseñanza que utilizó el caso de estudio. El inicio de su actividad dentro del aula se realiza con puntualidad, manteniendo un clima de respeto y disciplina en el salón. Comienza con un saludo y el establecimiento de normas y pautas, como por ejemplo: "cuando el profesor está explicando no deben revisar el libro, porque a la final no van a atender al libro, ni van a atender al profesor". Posteriormente da inicio a la explicación del tema, haciendo uso del pizarrón como su único recurso. A continuación, se hace la descripción según las categorías apriorísticas establecidas.

\section{Fundamento}

Con relación a la claridad de los objetivos, el docente lo declara: "el objetivo es conocer ¿cómo 
funcionan los modelos exponenciales?, ¿qué son?, ¿cómo funcionan y qué aplicaciones tienen?" lo hace de forma verbal, pero sin hacer mucho hincapié en ello; lo dice incluso de espalda hacia los estudiantes, y con una actitud tímida, sin verificar que sus aprendices lo internalizaran. Al considerar la propiedad recurrencia al programa o libro de texto, no se observa que el docente haga uso de ninguno de ellos durante el desarrollo de la clase, por el contrario, deja establecido al principio que sólo deben estar atentos a su explicación; este comportamiento es propio de un docente conductista, donde todo el conocimiento es poseído y transmitido por él. Al respecto, Ortíz (2013) afirma que "según los conductistas, para que los estudiantes aprendan basta con presentar la información" (p.10).

Respecto a la concentración en los procedimientos, esta es una de las propiedades más trabajadas, en el sentido de que el docente hace énfasis en el contenido procedimental de la función exponencial, en su graficación y la construcción de la tabla de valores. Esta propiedad está vinculada a la exhibición del conocimiento del tema, en este sentido el docente hace una presentación oral y escrita sobre el tema, sus fundamentos teóricos y procedimentales, donde deja en evidencia, mediante la exposición magistral, su dominio del conocimiento relacionado con la temática estudiada. Al igual que con el análisis de la propiedad anterior, el docente muestra ser un trasmisor de conocimientos, controlador y con poco o nula espontaneidad; conductas que lo ubican en el enfoque de enseñanza conductista según Ortíz (2013).

Con relación al uso de la terminología matemática se observó que hizo un buen uso. El concepto de función exponencial lo expresa como modelo, dentro de su análisis se notó que inició con la revisión de algunas propiedades de la potenciación según el exponente natural, entero, racional o irracional. Explicó las condiciones para la existencia de la operación $f(x)=a^{x}$, y estableció las condiciones de validez de su uso; es decir $a>0$ y $a \neq 1$ con dominio real.

\section{Transformación}

Esta categoría comienza con la propiedad selección de ejemplos. Aquí explicó a sus estudiantes un análisis de la función a partir del gráfico de la función $f(x)=2^{x}$, dando valores para la variable de $-2,-2,0,1,2$ y 3 , realizó en el pizarrón la tabla y gráfica correspondiente, luego de esto hizo preguntas como: “¿qué sucede si la variable toma un valor de 10?" ante lo cual, los estudiantes usando su calculadora responden: "da 1.024", y el docente comenzó a establecer una relación con los kilobytes, megabytes, gigabytes, terabytes. Convirtiendo de esta forma un ejemplo en una oportunidad para la vinculación con otra área de conocimiento, tal como la informática.

La propiedad selección de representaciones se notó cuando el docente convirtió la representación de la función ejemplificada $\left(f(x)=2^{x}\right)$, del registro algebraico al numérico, en este caso particular, se deben reconocer a las letras $x$ e y que configuran la expresión de origen como variables numéricas; $y$ al par numérico obtenido, se debe reconocer como un punto del plano para conseguir la representación gráfica de la función.

En cuanto a uso de material de enseñanza, este docente sólo utiliza el pizarrón, ningún otro material didáctico dentro del aula de clases, llama la atención que al principio de la clase, cuando 
da las orientaciones iniciales, dice a sus estudiantes: "el libro es un recurso para que usted lea la clase con anticipación, si es posible, de esta manera vendrá con dos cosas, con conocimiento y con dudas", posteriormente dice: "ahora, si usted no quiere leer la clase previo a que el profesor la dicte, puede igual consultar en ella luego de que el profesor haya dado la explicación", también hace énfasis en que "no deben usar la portátil", esto hace notar su poca inclinación positiva hacia las tecnologías de la información y la comunicación. Aunado a esto, hace uso casi exclusivo de la demostración por su parte, para enseñar un procedimiento.

\section{Conexión}

En cuanto a la conexión realizada por el docente entre la función exponencial como objeto matemático y otras disciplinas, la conexión entre procedimientos y conceptos y el reconocimiento de la adecuación conceptual, se consideran también las de tipo intraconceptual, interconceptual, temporal, interdisciplinar y extracurricular propuestas por Parra-Sandoval (2018).

El docente establece una relación o conexión entre la función exponencial y la informática, destacando un tipo de conexión interdisciplinar, esto es, exhibe la relación de un concepto matemático (función exponencial) con otras disciplinas del currículo escolar (informática). Esto lo hace al explicar mediante el ejemplo presentado de $f(x)=2^{x}$, cuando $x$ toma el valor de 10 , al sustituir, se obtiene para $\mathrm{f}(\mathrm{x})$ el valor de 1024 y esto lo conecta con la relación existente entre el kilobyte y el byte; entre el megabyte y el kilobyte y así sucesivamente. Sólo da esta conexión de la función con otra disciplina, dejando a un lado la posibilidad de conexión con la biología, demografía, economía, entre otras.

Con este ejemplo presentado por el docente también se evidencian las conexiones extracurriculares e intraconceptual, debido a la conexión de la función exponencial con aquellos conocimientos propios de una comunidad de prácticos (informáticos) y los conocimientos cotidianos (el kilobyte, el byte y el megabyte).

Con relación al vínculo entre procedimientos y conceptos, el docente conecta el tema función exponencial tanto conceptual como procedimentalmente con la potenciación, reconociendo de esta forma la adecuación conceptual de estos temas y la conexión de tipo interconceptual. Aquí se puede notar la conexión de tipo temporal, ya que hace una vinculación con el tema de potenciación estudiado en años anteriores según el currículo escolar ecuatoriano.

\section{Contingencia}

Tiene estructurado un plan de trabajo del cual no se desvía, actúa con gran organización. Da respuesta oportuna a las ideas e inquietudes de sus estudiantes, pero se nota que no hace uso de las oportunidades, la percepción que se tuvo del profesor durante la clase es de control, de autoridad exclusiva, además que es ajeno a la tecnología.

\section{Conclusiones}

Como consideración final e implicaciones derivadas de esta investigación, se tiene que la forma 
tradicional conductista de realizar la praxis pedagógica, caracterizada por el trabajo mecánico y repetitivo al enseñar conocimientos específicos aún se puede apreciar en Ecuador, así lo evidencian los resultados, en concordancia con lo de Avendaño (2013); Bravo, Trelles y Barrazueta (2017) y Suárez (2019). Es importante resaltar que la conexión de la función exponencial con otras disciplinas no se logró del todo, dejando a un lado la importancia de la vinculación de este tema con muchas actividades humanas relacionadas con la economía, biología, física, química, demografía, entre otras.

Al describir el proceso de enseñanza del tema tratado en una institución del Cantón Santo Domingo de los Colorados en Ecuador se concuerda con lo expuesto por Parra-Sandoval (2018), quien opina que es necesario explorar el trabajo interdisciplinario como espacio privilegiado para ampliar el conocimiento profesional que permite al profesor conectar un conocimiento matemático con otros conocimientos y saberes u otras áreas curriculares.

Los resultados de este estudio vislumbran la necesidad urgente de promover cambios en la praxis educativa de los docentes en ejercicio, lo cual puede ser a través de procesos de formación efectivos, con seguimiento y acompañamiento, lo cual representa un reto debido a que esto implica el desarrollo de competencias profesionales que le permitan al profesor de matemáticas vincular las matemáticas con otras realidades, esto conlleva a que se propongan nuevas formas de dinamizar e intervenir en ambientes escolares con temas matemáticos que pueden vincularse a algunas actividades humanas y así lograr que sus estudiantes se interesen más por lograr aprendizajes sobre las funciones exponenciales, como tema de gran importancia por sus aplicaciones a otros campos. 


\section{Referencias}

Avendaño, W. (2013). Un modelo pedagógico para la educación ambiental desde la perspectiva de la modificabilidad estructural cognitiva. Revista Luna Azul, 36, 110-133

Bravo, F.; Trelles, C. y Barrazueta. J. (2017). Reflexiones sobre la evolución de la clase de matemáticas en el bachillerato Ecuatoriano. Revista de la Universidad Internacional del Ecuador INNOVA, 2 (7), 1-12

Cantoral, R., Farfán, R., Cordero, F., Alanís, J. y Garza, A. (2003). Desarrollo del pensamiento matemático. México: Trillas

Castro, W.; Pino-Fan, L. y Parra-Urrea, Y. (2018). El Modelo del Conocimiento Didáctico-Matemático de los profesores: Nuevas perspectivas y horizontes para la formación docente. Revista Colombiana de Matemática Educativa, 3 (2), 18- 25

Garii, B. y Silverman, F. (2009). Beyond the classroom walls: Helping Teachers Recognize Mathematics Outside of the School. Revista latinoamericana de investigación en matemática educativa, 12 (3), 333-354.

Gómez, P. (2007). Desarrollo del conocimiento didáctico en un plan de formación inicial de profesores de matemáticas de secundaria. Granada: Departamento de Didáctica de la Matemática, Universidad de Granada

Husén, T. (1988). Paradigmas de la investigación en educación. En: I. Dendaluce. Aspectos metodológicos de la investigación educativa. Il Congreso mundial Vasco.

Martínez, P. (2006). El método de estudio de caso: estrategia metodológica de la investigación científica. Revista Pensamiento y Gestión, 20, 165-193

Monaghan, J. (2007). Linking School Mathematics to Out-of-school Mathematical Activities: Student Interpretation of Task, Understandings and Goals. International Electronic Journal of Mathematics Education, 2 (2), 50-71

Ortíz, A. (2013). Modelos Pedagógicos y Teorías del Aprendizaje ¿Cómo elaborar el modelo pedagógico de la institución educativa? Recuperado de https://www.researchgate.net/publication/315835198

Parra, H. (2013). Claves para la contextualización de la matemática en la acción docente. Revista Omnia, 19 (3), 74-85

Parra-Sandoval, H. (2018). La interdisciplinariedad como espacio para el desarrollo del horizonte matemático en profesores en ejercicio, Conferencia dictada en el I Congreso Virtual Iberoamericano sobre Formación de Profesores de Matemática, Ciencias y Tecnología (I CONVIBE FOR PRO). Noviembre, 2018

Quintana, A. y Montgomery, W. (Eds.) (2006). Psicología: Tópicos de actualidad. Lima: UNMSM 
Rowland, T., Huckstep, P., y Thwaites, A. (2005). Elementary teachers' mathematics subject knowledge: The knowledge quartet and the case of Naomi. Journal of Mathematics Teacher Education, 8 (3), 255-281

Shulman, L. (1986). Those who understand: Knowledge growth in teaching. Educational Researcher, 15 (2), 4-14

Shulman, L. (1987). Knowledge and teaching: Foundations of the new reform. Harvard educational review, 57 (1), 1-23

Suárez, L. (2019). Desempeño docente y rendimiento académico en el área de Matemática de la Institución Educativa "Carlos Julio Arosemena Tola" del Cantón de la provincia del Guayas Ecuador 2018. Tesis para optar el Grado Académico de Magíster en Educación con mención en Evaluación y Acreditación de la Calidad de la Educación. Universidad Nacional Mayor de San Marcos. Perú. 
Copyright (c) 2020 Carlos Armando Tarira Caice, Hugo Parra-Sandoval y Mercedes Delgado González

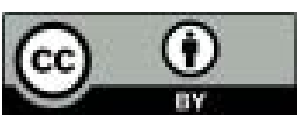

Este texto está protegido bajo una licencia internacional Creative Commons 4.0.

Usted es libre para Compartir-copiar y redistribuir el material en cualquier medio o formato

- y Adaptar el documento - remezclar, transformar y crear a partir del material-para cualquier propósito, incluso para fines comerciales, siempre que cumpla las condiciones de Atribución. Usted debe dar crédito a la obra original de manera adecuada, proporcionar un enlace a la licencia, e indicar si se han realizado cambios. Puede hacerlo en cualquier forma razonable, pero no de forma tal que sugiera que tiene el apoyo del licenciante o lo recibe por el uso que hace de la obra.

\section{$\underline{\text { Resumen de licencia - Texto completo de la licencia }}$}

